\title{
Pancreatic cancer cells surviving gemcitabine treatment express markers of stem cell differentiation and epithelial-mesenchymal transition
}

\author{
KARL QUINT $^{1}$, MANUEL TONIGOLD ${ }^{1}$, PIETRO DI FAZIO ${ }^{1}$, ROBERTA MONTALBANO ${ }^{1}$, \\ SUSANNE LINGELBACH ${ }^{1}$, FELIX RÜCKERT ${ }^{3}$, BEATE ALINGER ${ }^{2}$, \\ MATTHIAS OCKER ${ }^{1}$ and DANIEL NEUREITER ${ }^{2}$ \\ ${ }^{1}$ Institute for Surgical Research, Philipps University of Marburg, D-35043 Marburg, Germany; \\ ${ }^{2}$ Institute of Pathology, Paracelsus Private Medical University, A-5020 Salzburg, Austria; \\ ${ }^{3}$ Department of Surgery, University Clinic Mannheim, D-68167 Mannheim, Germany
}

Received July 13, 2012; Accepted September 3, 2012

DOI: 10.3892/ijo.2012.1648

\begin{abstract}
Objective response rates to standard chemotherapeutic regimens remain low in pancreatic cancer. Subpopulations of cells have been identified in various solid tumors which express stem cell-associated markers and are associated with increased resistance against radiochemotherapy. We investigated the expression of stem cell genes and markers of epithelial-mesenchymal transition in pancreatic cancer cells that survived high concentrations of gemcitabine treatment. Capan-1 and Panc-1 cells were continuously incubated with 1 and $10 \mu \mathrm{M}$ gemcitabine. Surviving cells were collected after 1, 3 and 6 days. Expression of PDX-1, SHH, CD24, CD44, CD133, EpCAM, CBX7, OCT4, SNAIL, SLUG, TWIST, Ki-67, E-cadherin, $\beta$-catenin and vimentin were quantified by qPCR or immunocytochemistry. Migration was assessed by wound-healing assay. SHH was knocked down using RNA interference. Five primary pancreatic cancer cell lines were used to validate the qPCR results. All investigated genes were upregulated after 6 days of gemcitabine incubation. Highest relative expression levels were observed for OCT4 (13.4-fold), CD24 (47.3-fold) and EpCAM (15.9-fold) in Capan-1 and PDX-1 (13.3-fold), SHH (24.1-fold), CD44 (17.4fold), CD133 (20.2-fold) and SLUG (15.2-fold) in Panc-1 cells. Distinct upregulation patterns were observed in the primary cells. Migration was increased in Panc-1 cells and changes in the expression of E-cadherin and $\beta$-catenin were typical of epithelial-mesenchymal transition in both cell lines. $\mathrm{SHH}$ knockdown reduced $\mathrm{IC}_{50}$ from 30.1 to $27.6 \mathrm{nM}$ in Capan-1 while it strongly inhibited proliferation in Panc-1 cells. Cells surviving
\end{abstract}

Correspondence to: Dr Karl Quint, Institute for Surgical Research, Philipps University Marburg, Baldingerstrasse, D-35043 Marburg, Germany

E-mail:karl.quint@med.uni-marburg.de

Key words: chemoresistance, differentiation, embryonic, gemcitabine, epithelial-mesenchymal transition, pancreatic cancer high-dose gemcitabine treatment express increased levels of stem cell genes, show characteristics associated with epithelialmesenchymal transition and retain their proliferative capacity.

\section{Introduction}

Pancreatic cancer is among the malignancies with the worst patient outcome, representing the fourth most common cause of cancer-related death in the US $(1,2)$. Unfortunately, at the time of diagnosis, only a minority of tumors are restricted locally and therefore resectable, while the majority ( $>85 \%$ ) already shows regional or distant spread. For these late stage tumors, systemic chemotherapy with antimetabolites such as 5-fluorouracil (5-FU) or gemcitabine is only a palliative option. Although gemcitabine has shown improved objective response rates over 5-FU (3) in terms of tumor mass reduction and time to progression, most studies investigating gemcitabine-based combinations have failed to show a statistically significant survival benefit compared to gemcitabine alone. An exception is the combination of gemcitabine with the tyrosine kinase inhibitor erlotinib, for which an increase of $6 \%$ in one-year survival rates over gemcitabine alone could be achieved. However, the median survival improvement was merely ten days and no statistically significant objective response rates compared to gemcitabine monotherapy were achieved (4). The recent FOLFIRINOX study reports median overall survival rates of about $10 \%$, however, these rates still lag behind achievable rates in other gastrointestinal tumors (5). Overall, poor response rates after single agent and combined therapies as well as the development of chemoresistance result in a disappointing 5-year median survival rate of $5 \%$, a number that has not changed significantly during the past 40 years $(1,6,7)$.

A characteristic feature of pancreatic cancer is its intrinsic resistance to chemotherapy, which is mediated by various factors, such as hypovascularization, prominent desmoplasia, the expression of drug metabolizing enzymes, and, as recent publications suggest, the presence of putative pancreatic cancer stem cells (8-10). The concept of organ stem cells, slow-cycling cells with the capacity of unlimited self-renewal, asymmetric 
cell division and differentiation into mature cell types which reside in a supportive microenvironment/niche, is widely accepted (5,9,11-15). In the context of cancer, it implicates that cell types within a tumor are unequal at any given time and that there is a predetermined cell population with a stem cell phenotype, which perpetuates the tumor while other cells of the same cancer are incapable of self-renewal. It has also been shown that the cancer stem cell subpopulation exhibits an enhanced resistance to chemotherapy and radiation, both in hematologic malignancies and solid tumors $(16,17)$ and cell lines $(18,19)$. At present, however, the exact stem cell phenotype characterizing this subpopulation of cells is not known for most solid tumors. $\mathrm{CD} 24^{+} \mathrm{CD} 44^{+} \mathrm{ESA}^{+}$or $\mathrm{CD} 133^{+} \mathrm{CXCR} 4^{+}$cells have been proposed to represent this subpopulation in pancreatic cancer based on their ability to self-renew, to effectively form xenografts in nude mice or being able to metastasize (20). Side populations, defined as cells that are able to exclude DNA-binding dyes such as Hoechst 33342, have also been shown to be rich in $\mathrm{CD} 133^{+}$ cells and also express higher levels of drug-efflux pumps such as $\mathrm{ABCG} 2$, which have been associated with gemcitabine resistance (21). Besides the pre-existence of inherently resistant stem cells, several studies showed that the epithelial-mesenchymal transition (EMT) is another mechanism that is triggered when cells are challenged by cytostatic drugs (22-24). In the context of cancer, tumor cells of epithelial origin revert to a mesenchymal state, survive chemotherapy and acquire an enhanced migratory and invasive potential that might be responsible for tumor recurrence and metastasis. Interestingly, when EMT is induced in tumor cells, not only the presence of transformed mesenchymal cells increases, but also the presence of cells with the above described cancer stem cell phenotype (25). Taken together, these studies indicate that the stem cell phenotype and the EMT process are functionally linked and might confer resistance to chemotherapy.

We therefore hypothesized that high-dose gemcitabine treatment would enrich chemotherapy resistant cells which can be identified by their expression of stem cell associated genes. Here we investigate the expression of several known and new stem cell markers along with EMT-associated genes in response to high-dose gemcitabine treatment and show that surviving cells express increased levels of stem cell genes and acquire the molecular characteristics typical for EMT.

\section{Materials and methods}

Cell culture. Panc-1 cells were grown in DMEM (Biochrom, Berlin, Germany) substituted with $10 \%$ fetal bovine serum (FBS, Biochrom). Capan-1 cells were maintained in RPMI (Biochrom) with 20\% FBS. All media contained 1\% penicillin/streptomycin and $0.5 \%$ gentamicin (Biochrom). Cells were grown in culture flasks (Nunc, Langenselbold, Germany) under standard culture conditions $\left(37^{\circ} \mathrm{C}, 5 \% \mathrm{CO}_{2}\right)$. Medium was changed every 3 days. Passages 6 to 18 were used for all experiments. Cells tested negative for Mycoplasma infection. Primary pancreatic cancer cells PaCaDD135, -159, -161, -165 and -188 were established and cultured as described previously $(26,27)$.

Drug preparation. Gemcitabine was obtained from GRY Pharma (Kirchzarten, Germany). Fresh stock solutions were prepared in concentrations of $144 \mathrm{mM}$ in phosphate-buffered saline (PBS, Biochrom), kept in aliquots at $-20^{\circ} \mathrm{C}$ and diluted in medium to the final concentrations.

Viable cell counting. Of each cell line, $10^{5}$ cells/well were seeded in 6-well plates and allowed to attach overnight. Medium was removed, wells washed with PBS once and new medium added to the wells, containing gemcitabine in final concentrations of 0.01 to $100 \mu \mathrm{M}$. Untreated controls were run in parallel. At each time-point of analysis, cells were washed, trypsinized using trypsin/EDTA solutions (Biochrom) and resuspended in medium. Cells were counted after trypan blue staining in a Neubauer chamber. Experiments were performed in triplicates.

RNA purification and reverse transcription. For the extraction of total cellular RNA, the medium was removed from the culture flasks and the cell layer was washed with PBS prior to adding trypsin. The trypsinized cells were resuspended in medium and counted. Cells $\left(1.5 \times 10^{6}\right)$ were centrifuged at $1,000 \mathrm{x}$ g for $10 \mathrm{~min}$ and the cell pellet was subsequently used for RNA purification with RNeasy mini kit (Qiagen, Hilden, Germany) according to the manufacturer's instructions. RNA concentration was determined photometrically on an Eppendorf BioPhotometer (Eppendorf, Hamburg, Germany). RNA (1 $\mu \mathrm{g})$ was used for cDNA synthesis using the iScript cDNA Synthesis kit (Bio-Rad, Munich, Germany) following the manufacturer's instructions.

Quantitative real-time PCR. Quantitative real-time PCR (qPCR) was performed on a Bio-Rad CFX96 Real-Time System (Bio-Rad Laboratories, Munich, Germany) using SsoFast EvaGreen Supermix (Bio-Rad) and QuantiTect Primer Assays for SHH, PDX1, GLI, PTCH, SMO, NOTCH, CD24, CD44, CD133, EpCAM, SNAI1 (Snail), SNAI2 (Slug), TWIST, OCT4, CBX7 and GAPDH (all from Qiagen) following the manufacturer's standard procedure outlined in the SYBR-Green leaflet. Reaction efficiency was determined using standard curves. Gene expression analysis was computed using CFX Manager 2.0 (Bio-Rad) and REST 2009 (Qiagen). To indicate the baseline expression of each gene of interest in untreated cells, the $\Delta \mathrm{C}_{\mathrm{t}}$ value was calculated as follows: $\Delta \mathrm{C}_{\text {tgene of interest }}=$ $\mathrm{C}_{\text {tgene of interest }}-\mathrm{C}_{\mathrm{tGAPDH}}$, whereby high $\Delta \mathrm{C}_{\mathrm{t}}$ values indicate low baseline expression. Changes in gene expression comparing treated with untreated cells were computed using the $2^{-\Delta \Delta \mathrm{Ct}}$ method with GAPDH as reference gene and expressed as efficiency corrected $n$-fold changes \pm standard error of the mean (SEM). Measurements were performed in triplicates. Statistical significant changes in gene expression were assessed using the algorithm within the REST 2009 software $(28,29)$ and a p-value of $<0.05$ was considered statistically significant.

Preparation of cell blocks and cover slips. Cell pellets were resuspended in $200 \mu \mathrm{l}$ citrate plasma and $200 \mu \mathrm{l}$ Thromborel S (Siemens Healthcare Diagnostics, Deerfield, IL, USA) was added. After coagulation, cells were fixed for $1 \mathrm{~h}$ in neutral-buffered saline containing $7 \%$ formalin and were paraffin-embedded. In cases where morphology was to be preserved, cells grown on coverslips were used. Fifty thousand cells per well were plated in 24-well plates on round glass cover slips (Thermo Scientific, Braunschweig, Germany), allowed to attach overnight before treatment was performed as indicated. 
At each respective time-point, cells were washed with PBS and fixed with acetone/methanol $(1: 2 \mathrm{v} / \mathrm{v})$ and stored at $-20^{\circ} \mathrm{C}$ until immunocytochemical staining.

Immunocytochemical staining. Cell blocks were cut into 5- $\mu \mathrm{m}$ sections and deparaffinized using graded alcohols. Antigen retrieval was performed by heat-induced epitope retrieval in pH 9.0 antigen retrieval buffer (Dako, Glostrup, Denmark) at $95^{\circ} \mathrm{C}$ for $60 \mathrm{~min}$. Endogenous peroxidase blocking was carried out for $10 \mathrm{~min}$ with peroxidase-blocking reagent (Dako). Subsequently, mouse monoclonal primary antibodies against $\beta$-catenin (1:200, Dako), E-cadherin (1:100, NeoMarkers), vimentin (1:2000, Dako) and Ki-67 (1:500, Dako) were applied for $30 \mathrm{~min}$ at RT. Primary rabbit and mouse antibodies were detected using the EnVision Detection System (Dako). Staining of the coverslips was performed against SHH (rabbit monoclonal, 1:100), CD133 (rabbit polyclonal, 1:100), OCT4 (rabbit polyclonal, 1:80) and PDX-1 (rabbit polyclonal, 1:3000) (all from Abcam, Cambridge, UK) and diluted in background reducing antibody diluent (Dako), with Vectastain Elite ABC Kit Rabbit (Vector Laboratories, Burlingame, CA, USA) adhering to the manufacturer's instructions. Visualization was performed using DAB (Roche, Mannheim, Germany) and counterstained with haematoxylin. Control experiments for all stainings were negative using PBS replacement of primary or secondary antibodies and same processing as described above. The stained slides were digitalized using the ImageAccess 9 Enterprise software (Imagic Bildverarbeitung, Glattbrugg, Switzerland) and percentage of positive cells evaluated using the Cell Explorer 2006 software (BioSciTec, Frankfurt, Germany). In addition, staining intensities were graded semiquantitatively as negative, low, moderate and high.

In vitro wound healing (scratch) assay. Panc-1 cells $\left(10^{5}\right.$ well) were seeded into 6-well plates and allowed to grow to approximately $80 \%$ confluence. One day prior to the application of the scratch, gemcitabine was added to the wells to a final concentration of $10 \mu \mathrm{M}$. PBS was used as control. After $24 \mathrm{~h}$ of incubation, a scratch was applied to the cell layer across each well using a $200 \mu \mathrm{l}$ pipette tip. The cell layer was washed twice with PBS to remove lose cells from the scratch margins. The wells were refilled with $2 \mathrm{ml}$ of fresh medium and gemcitabine added, except in the controls. At regular, intervals images were taken from definite locations of the scratches with a Nikon Coolpix 995 digital camera on a Zeiss Axiovert 40C phase contrast inverted microscope with scattered light illumination.

Time-lapse microscopy. Time-lapse recording was performed using the JuLi Smart Fluorescent Cell Analyzer (PAA Laboratories, Cölbe, Germany) under standard conditions.

RNA-interference. Gene knockdown experiments were performed in 6-well plates using the siLentFect Lipid Reagent (Bio-Rad) and siRNA against SHH (Hs_SSH_6, SI03080182) and control non-coding siRNA (AllStars Negative Control, 1027280 , both from Qiagen). Cells (1.5x105/well) were seeded 1 day prior to transfection and allowed to attach overnight. One hour prior to transfection, the medium was replaced with fresh antibiotics-free medium containing 1\% FCS. For each well to be transfected, $3 \mu \mathrm{l}$ siLentFect reagent was diluted in
$150 \mu 1$ OptiMEM I (Life Technologies, Darmstadt, Germany) and $3.6 \mu \mathrm{l}$ siRNA solution in $150 \mu \mathrm{l}$ OptiMEM. Diluted siRNA and diluted siLentFect were mixed and incubated at room temperature for $20 \mathrm{~min}$ after which they were slowly added to the respective wells. The cells were incubated overnight, after which they were trypsinized and used in the xCELLigence assay. A portion of the same cells was used for determining the knockdown efficiency by qPCR.

$x$ CELLigence assay. Impedance-based real-time measurement of cellular proliferation and $\mathrm{IC}_{50}$ calculation were performed on the xCELLigence Real-Time Cell Analyzer (RTCA) in designated 96-well electrode plates (E-plates) (all from Roche Applied Science, Penzberg, Germany) under standard culture conditions (30). The RTCA software v. 1.2 was used for data recording, analysis of proliferation and $\mathrm{IC}_{50}$ calculation. In all experiments, $50 \mu \mathrm{l}$ of cell-free medium was added to each well of the E-plate and background measurement performed. Next, $100 \mu \mathrm{l}$ of cell suspension $\left(10^{4}\right.$ cells $\left./ 100 \mu \mathrm{l}\right)$ were added to each well, measurement was started and the cells allowed at attach and proliferate for $24 \mathrm{~h}$ prior to the addition of the compound. Readings were performed every $15 \mathrm{~min}$ for at least 3 days. $\mathrm{IC}_{50}$ at day 3 was determined by incubating the cells with serial concentrations of gemcitabine ranging from 0.001 to $10 \mu \mathrm{M}$. The readout as recorded by the RTCA is expressed as a dimensionless cell index (CI) value which correlates with cell number and size. Measurements were performed in quadruplicates.

\section{Results}

Determination of optimal gemcitabine concentration. Aiming to analyze only the most resistant cells, we decided to find the optimal concentration that would allow $5 \%$ of the cells to survive 3 days of continuous gemcitabine incubation. By treating Capan-1 and Panc-1 cells with serial concentrations of gemcitabine and counting surviving cells after 3 days, we determined that 1 and $10 \mu \mathrm{M}$, respectively, of gemcitabine were needed to kill $95 \%$ of the cells. After 6 days of treatment at the indicated concentrations, surviving cells could still be observed. Therefore, gene expression analysis was performed at time-points of 1,3 and 6 days of continuous gemcitabine incubation in order to capture time-dependent changes in gene expression. Capan-1 cells still viable after day 12 of $100 \mu \mathrm{M}$ gemcitabine could be observed.

mRNA expression of stem cell-and EMT-associated markers. The expression of all investigated markers was increased in the surviving cells after 6 days of continuous incubation with gemcitabine (Fig. 1). In the group of the stem-cell associated markers, PDX1 and SHH reached the highest expression after 6 days in Panc-1 cells (13.4 and 24.1-fold, respectively) and PDX1 and OCT4 in Capan-1 cells (4.1 and 13.4-fold, respectively). OCT4 and PDX1 in Capan-1 and Panc-1 cells, respectively, were also the genes most strongly induced already after 1 day of treatment (Table IA). In the group of tumor stem cell markers, CD44 and CD133 showed the highest increase in Panc-1 (17.4 and 20.2-fold, respectively), while CD24 and EpCAM demonstrated the highest expression in Capan-1 (47.3 and 15.9-fold, respectively). Interestingly, genes which reached the highest expression in Panc-1 (CD44 and CD133) showed only moderate expression levels in Capan-1, while reciprocally, the highest 

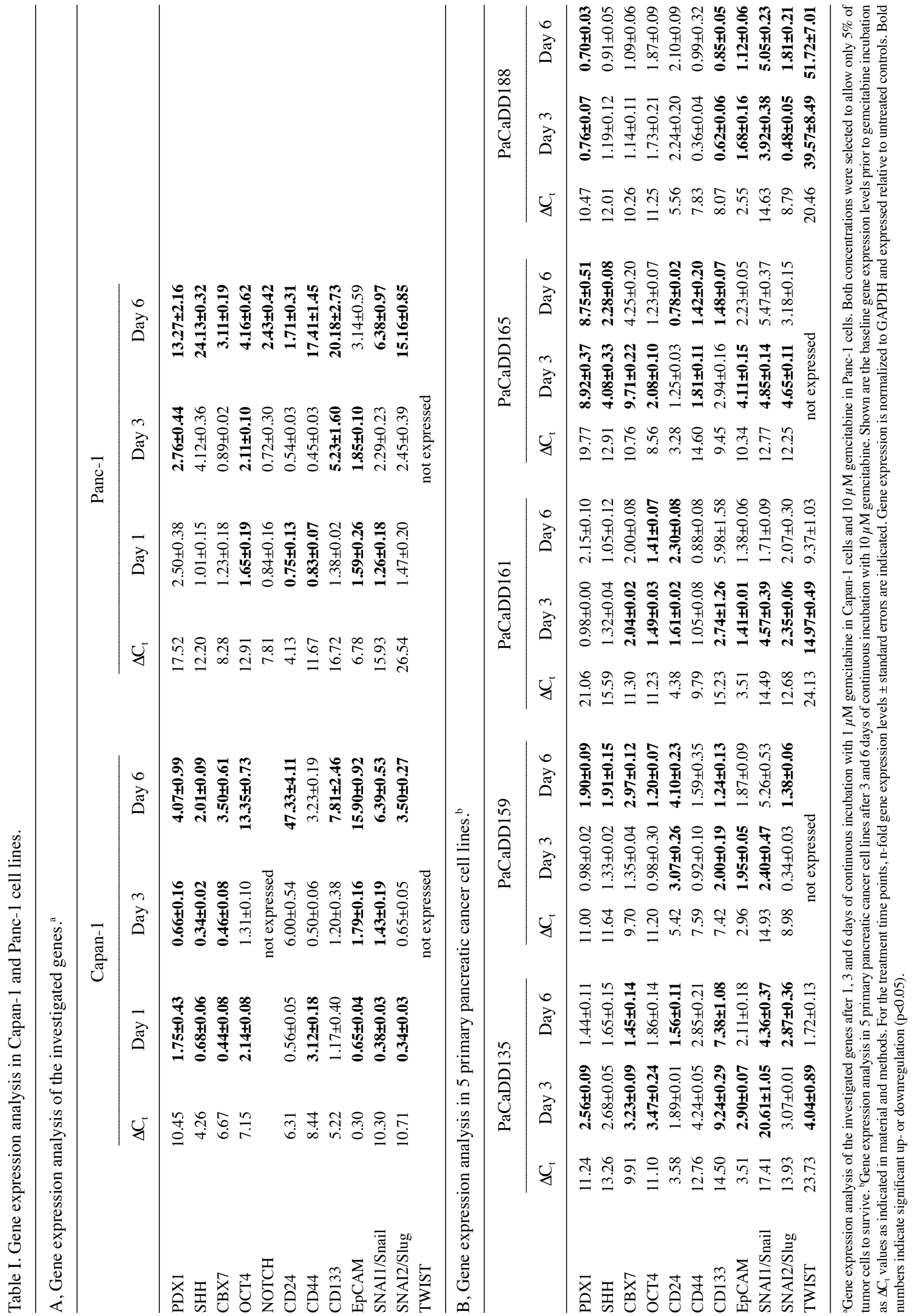

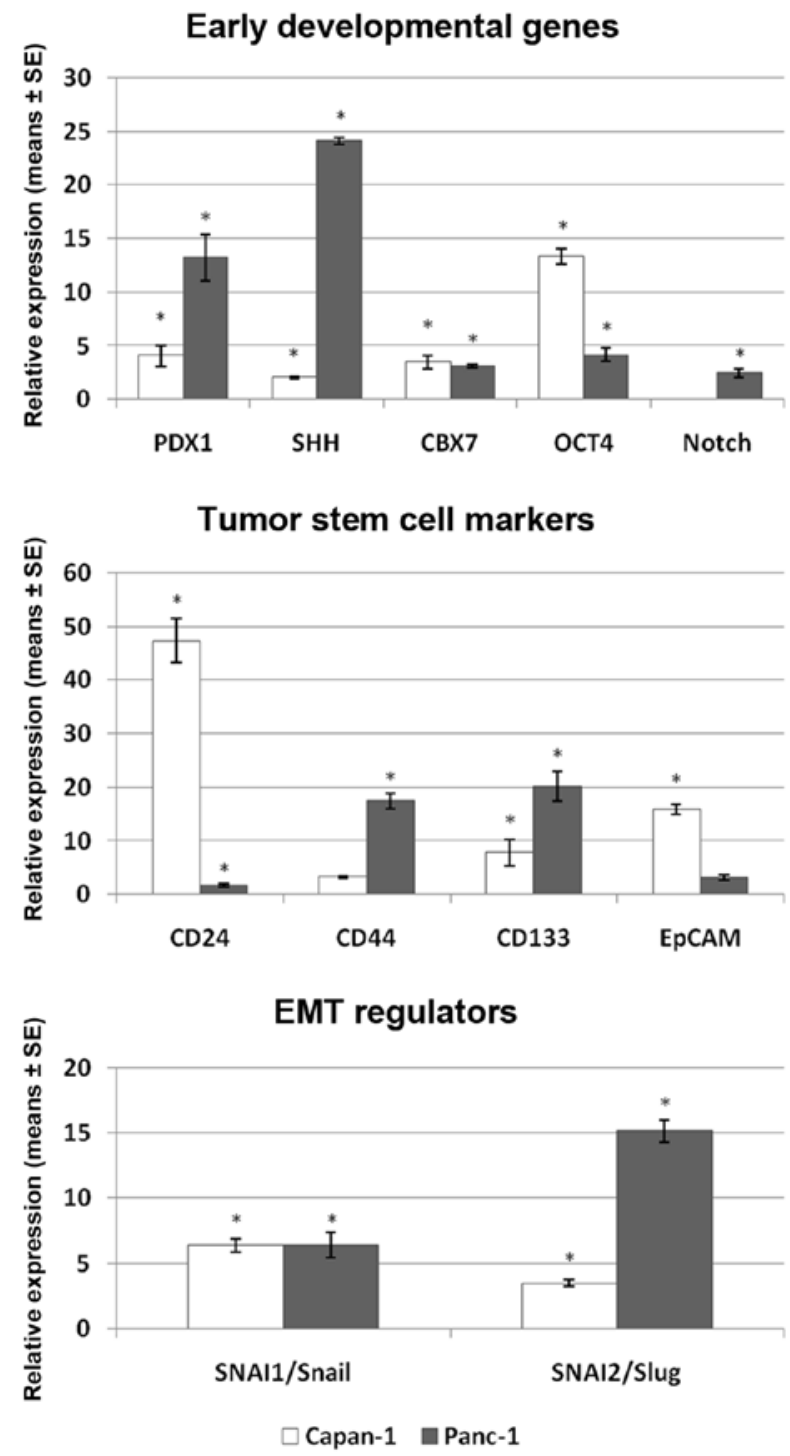

Figure 1. Relative gene expression changes in Capan-1 and Panc-1 cells surviving $1 \mu \mathrm{M}$ and $10 \mu \mathrm{M}$ continuous gemcitabine incubation for 6 days, respectively. Bars indicate $n$-fold relative gene expression. Expression is normalized to GAPDH and untreated controls, error bars indicate standard error, asterisks indicate significant changes $(\mathrm{p}<0.05)$.

expressed genes in Capan-1 (CD24 and EpCAM) reached only low to moderate levels in Panc-1 cells. The EMT regulators SNAI1/Snail and SNAI2/Slug showed a time-dependent increase in expression in both cell lines. Snail and Slug were induced 6.4- and 3.5-fold in Capan-1 and 6.4- and 15.2-fold in Panc- 1 cells after 6 days of continuous gemcitabine treatment. TWIST was not detectable in either of the two cell lines. A complete list of gene expression levels at 1, 3 and 6 days of treatment are listed in Table IA.

Immunocytochemical validation of gene expression. We performed immunocytochemical stainings for SHH, OCT4, PDX1 and CD133 in untreated cells and after 6 days of gemcitabine incubation to confirm the qPCR results (Fig. 2). Both untreated and treated Capan-1 cells expressed $\mathrm{SHH}$ in $100 \%$ of the cells. However, the gemcitabine treated cells showed a stronger staining intensity. Of note was that small colonies and single cells showed a stronger expression in contrast to larger colonies in both untreated and treated cells. PDX1 was nuclearly expressed in $30 \%$ of untreated Capan-1 cells and increased in foci number and intensity of staining in the nuclei of treated cells. OCT4 stained positive in granular fashion within the nuclei of $100 \%$ of untreated cells and to a greater extent both in number of foci and intensity in the treated cells. CD133 was expressed in $50 \%$ of the untreated Capan- 1 cells at low to moderate intensities. Occasionally, strong staining intensities were observed in singular cells or groups of 4-10 cells. In the treated group, all cells stained positive for CD133 and staining intensity reached moderate to high levels.

In untreated Panc-1 cells, SHH was expressed in $40 \%$ of the cells in moderate to strong staining intensity and these cells were arranged in groups. After gemcitabine incubation, $90 \%$ of the cells showed strong staining intensity. Nuclear staining for PDX1 was observed in $100 \%$ and cytoplasmic staining in $20 \%$ of untreated Panc- 1 cells. Treatment increased the number of nuclear foci, accompanied by a cytoplasmic reaction in $90 \%$ of the cells. Granular OCT4 positivity was observed in $100 \%$ of the nuclei of both untreated and treated cells and in the cytoplasm and perinuclear region of $30 \%$ of cells. Treatment with gemcitabine led to an increase in nuclear foci along with stronger staining intensities. CD133 was positive in $30 \%$ of untreated cells and expressed in a polar pattern with weak to moderate intensities. After treatment, $70 \%$ of the cells stained positive and CD133 was localized in the perinuclear region.

Validation of EMT. We demonstrated an induction of EMT markers by qPCR in Panc-1 and to a lesser extent also in Capan-1 cells after 6 days of gemcitabine treatment. We therefore investigated $\beta$-catenin and E-cadherin expression, and $40-50 \%$ of the untreated Panc- 1 cells expressed $\beta$-catenin and E-cadherin on the cell membranes (Fig. 3A). Continuous incubation with gemcitabine for 6 days resulted in a marked nuclear translocation of $\beta$-catenin and repression of membranous E-cadherin. Similar but less distinct patterns were observed in Capan-1 cells. Expression of vimentin, considered a marker of immature and mesenchymal phenotypes, was prominent in untreated Panc-1 cells and gemcitabine incubation did not further increase its expression. In Capan-1 cells, vimentin was present in untreated cells, while, interestingly, most cells showed a decrease in vimentin expression after gemcitabine incubation.

To determine if the surviving cells retained their proliferative capacity, Ki-67 was used to identify the cells undergoing DNA replication. Ki-67 expression was present in $86 \%$ of untreated Capan-1 cells and in $95 \%$ of the surviving cells after gemcitabine incubation. In untreated Panc- 1 cells, $46 \%$ were Ki-67 positive and $86 \%$ after gemcitabine incubation (Fig. 3B).

Wound healing assay. EMT is associated with loss of cell-cell adhesions and increased cell migration. We therefore performed a wound healing assay to assess cell migration in the gemcitabine treated Panc-1 cells, which showed the quickest and highest induction of Snail and Slug. Wound closure was achieved after 3.5 days after scratch application (corresponding to day 4.5 of gemcitabine treatment) due to an increased cell migration (Fig. 4). In contrast, the wound in the untreated cells was still clearly visible at this time. These findings were confirmed by time-lapse microscopy showing an increased undirected 

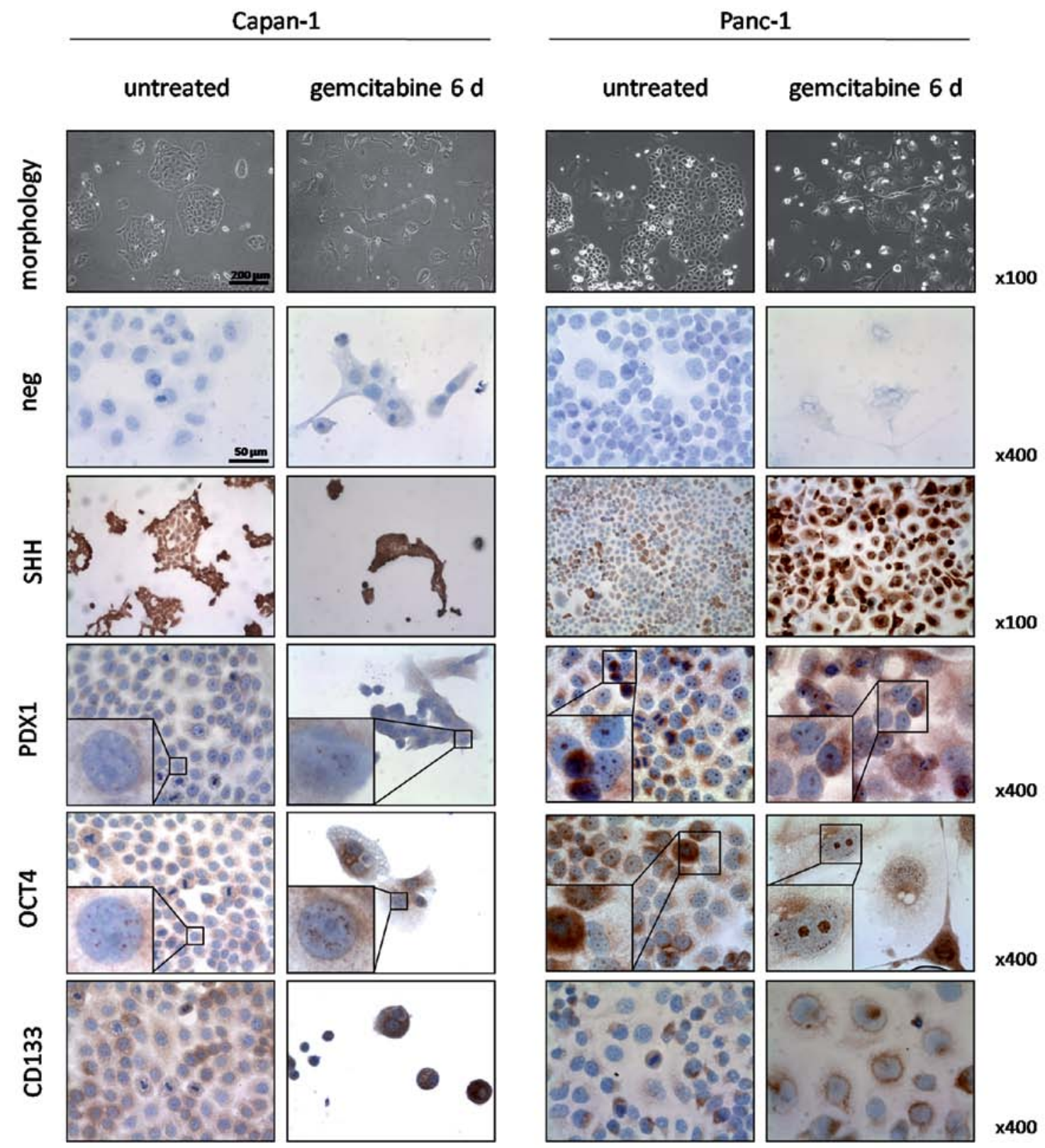

Figure 2. Immunocytochemical validation of gene expression. Shown are cell morphology, negative controls and stainings for SHH, PDX1, OCT4 and CD133 in Capan-1 and Panc-1 cells after 6 days of continuous gemcitabine incubation. Markers in which nuclear staining is present are also shown in magnified inserts. Magnifications of the panels, $\mathrm{x} 100$ and $\times 400$.

migration in treated cells starting from day 2 of treatment, while untreated controls showed little movement throughout the observation period (not shown).

siRNA mediated downregulation of SHH. The expression of SHH after 6 days of gemcitabine incubation showed the highest difference among the two investigated cell lines. Therefore, siRNA mediated knockdown of SHH was performed and its influence on $\mathrm{IC}_{50}$ values of gemcitabine was assessed using the xCELLigence system (Fig. 5 A, B, D and E). A knockdown ratio for siSHH below 33\% was achieved in both cases (Fig. 5, $\mathrm{C}$ and $\mathrm{F}$ ). $\mathrm{IC}_{50}$ of control siRNA transfected Capan-1 cells was $30.1 \mathrm{nM}$ (Fig. 5A) and siSHH transfection decreased $\mathrm{IC}_{50}$ to $27.6 \mathrm{nM}$ (Fig. 5B). In control siRNA transfected Panc-1 cells,
$\mathrm{IC}_{50}$ was $106.8 \mathrm{nM}$ (Fig. 5D). Interestingly, siSHH transfection revealed that proliferation in Panc-1 cells is dependent on $\mathrm{SHH}$ as the transfected cells did not show an increase in cell index (Fig. 5E). After 72-96 h, when the transient knockdown effect attenuated, the cells reverted to an increased proliferation rate, indicating a reversible inhibition of proliferation through transient $\mathrm{SHH}$ knockdown. Determining the $\mathrm{IC}_{50}$ in these cells was therefore not possible.

Expression of stem cell associated genes and EMT regulators in primary pancreatic cancer cell lines. To confirm our results in a further model which resembles patient tumors more closely, we performed the same treatment with $10 \mu \mathrm{M}$ gemcitabine in five recently established primary pancreatic cancer cell lines 
A
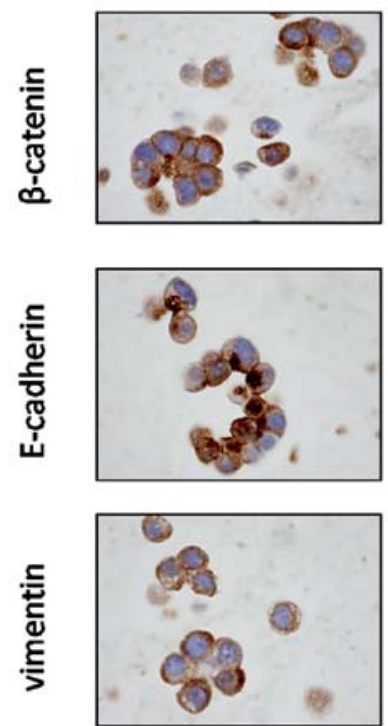

Capan-1

gemcitabine $6 \mathrm{~d}$
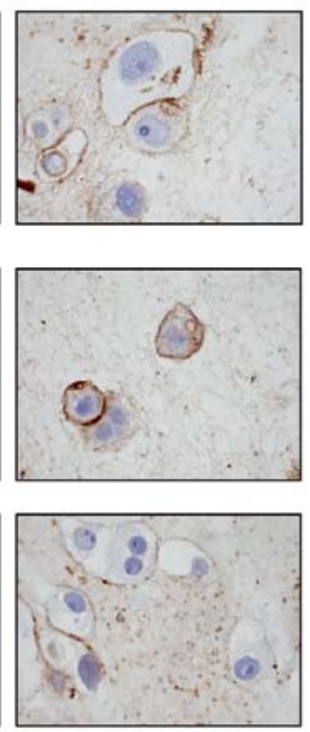

Panc-1
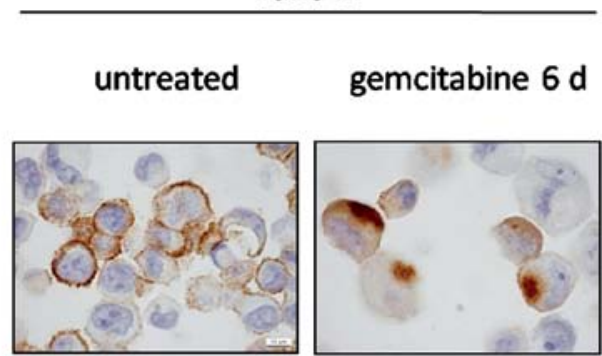

$\times 1000$
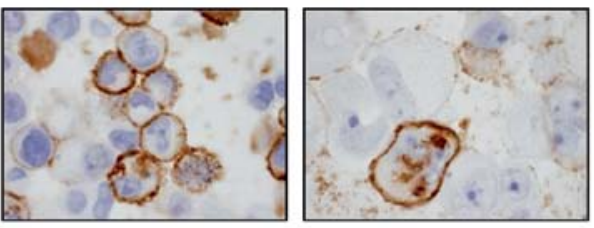

$\times 1000$
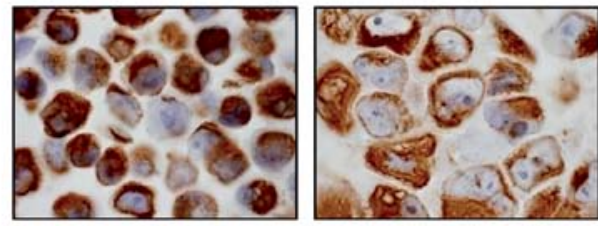

$\mathbf{x 1 0 0 0}$
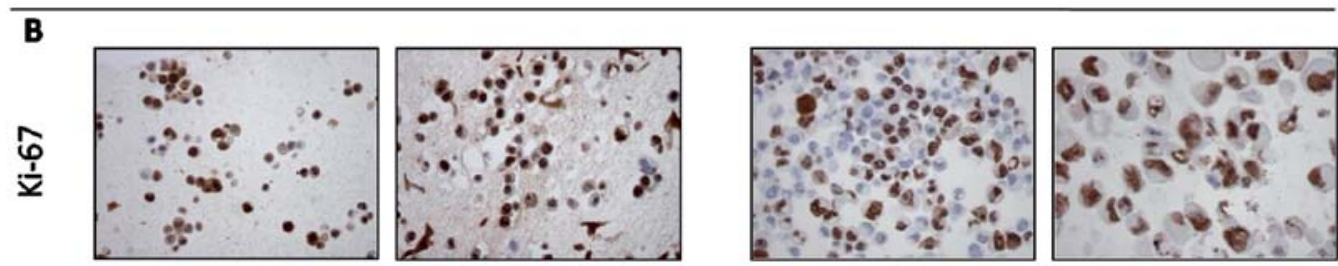

$\times 400$

Figure 3. (A) Immunocytochemical staining for EMT-associated proteins $\beta$-catenin, E-cadherin, vimentin and (B) proliferation marker Ki-67. Shown are untreated cells and cells after 6 days of continuous gemcitabine incubation. Magnifications of the panels, x1,000 and x400.

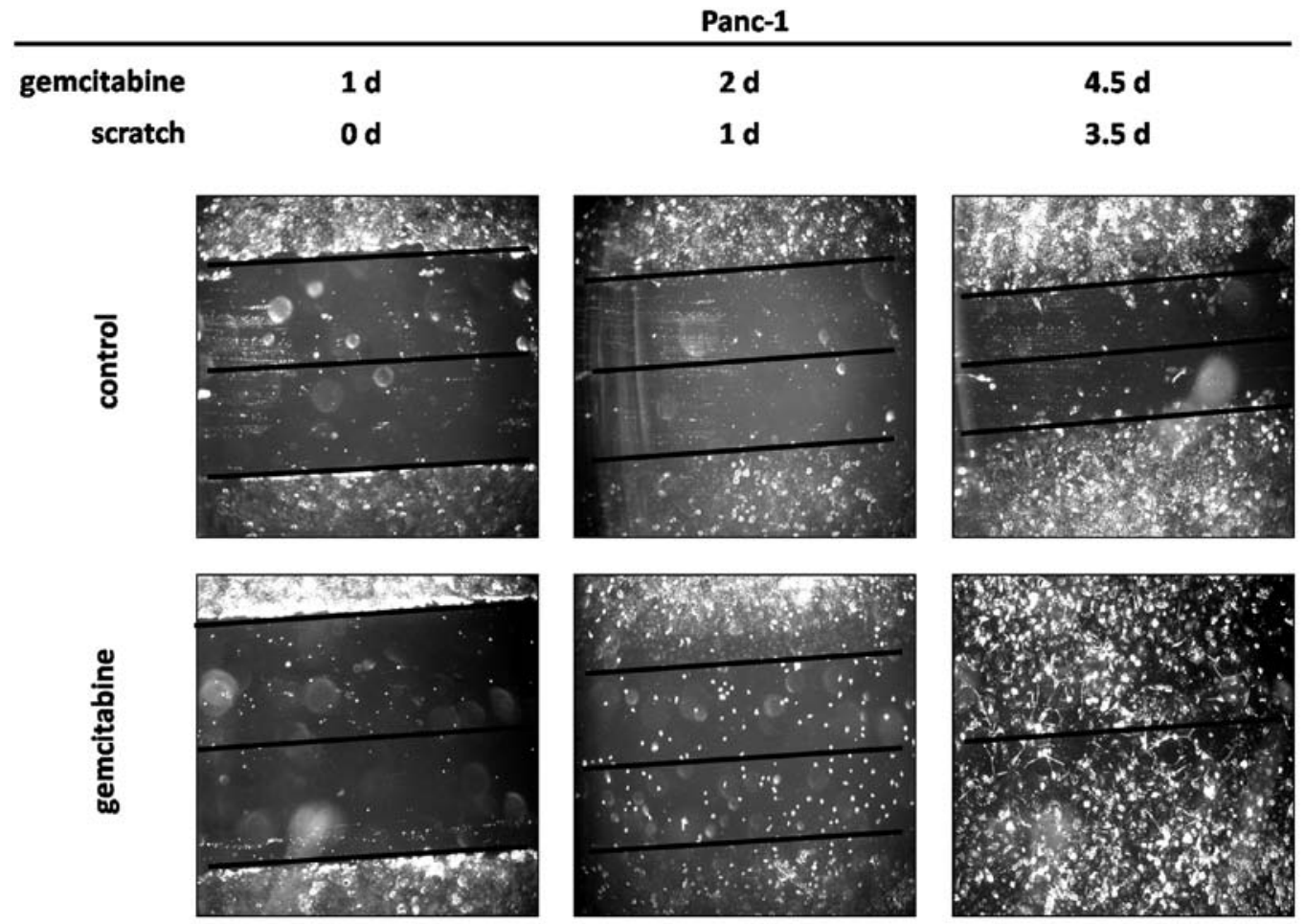

Figure 4. Scratch assay on gemcitabine treated cells in comparison with untreated controls. Gemcitabine incubation was initiated at a concentration of $10 \mu \mathrm{M}$ one day prior to applying the scratch. At day 3.5 after application of the scratch, the wound was closed in the treated while still clearly visible in the untreated cells. 

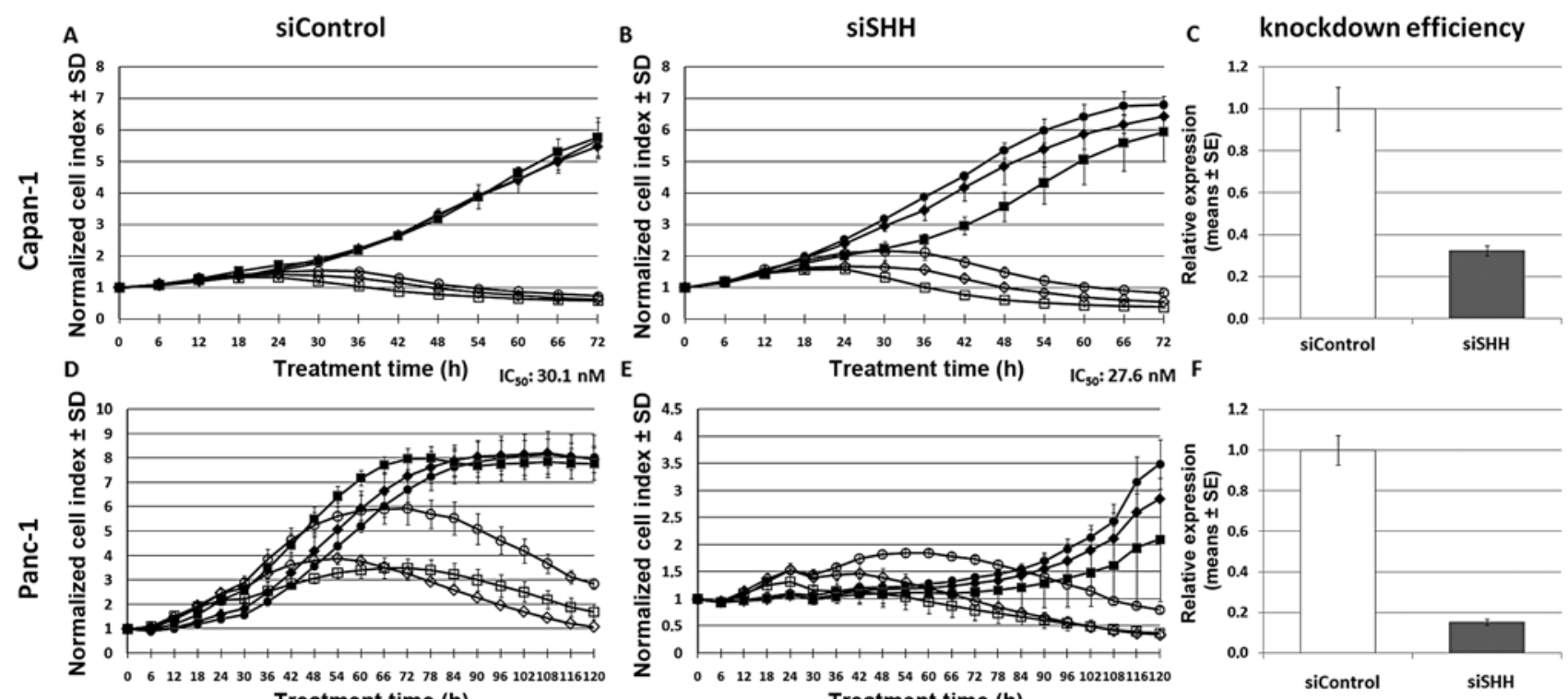

Treatment time (h) $\quad \mathrm{IC}_{\mathrm{so}}: 106.8 \mathrm{nM}$
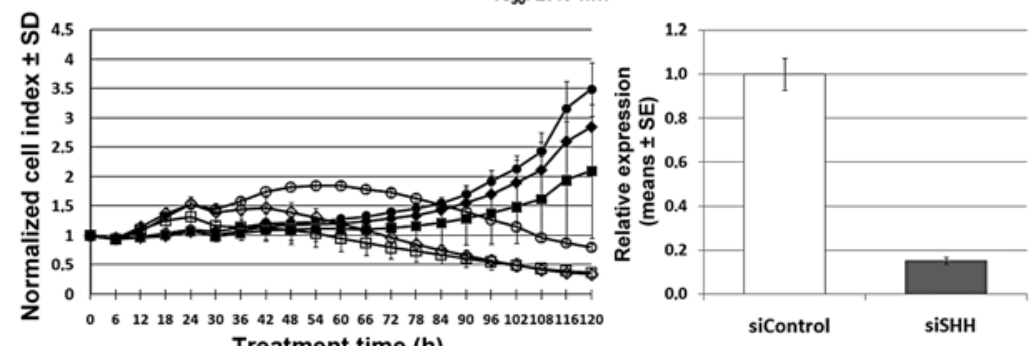

$\rightarrow-$ Control $\rightarrow 1 \mathrm{nM} \rightarrow-10 \mathrm{nM} \odot 100 \mathrm{nM} \nrightarrow 1 \mu \mathrm{M} \nsucc 10 \mu \mathrm{M}$

Figure 5. siRNA mediated knockdown of SHH in Capan-1 and Panc-1 cells and the influence on $\mathrm{IC}_{50}$. Both cell lines were transfected with control siRNA (siControl, panels A and D) and siRNA against SHH (siSHH, panels B and E) and subsequently seeded in 96-well plates in an impedance based cell analyzer system (xCELLigence Real Time Cell Analyzer). SHH knockdown efficiency was assessed using qPCR (panels C and F). Proliferation was measured under serial concentrations of gemcitabine and in untreated controls. Each data point represents the mean CI-value of quadruplicates \pm standard deviation (SD) in panels A, B, $\mathrm{D}$ and $\mathrm{E}$, and mean relative expression \pm standard error (SE) in panels $\mathrm{C}$ and $\mathrm{F}$. CI-values were normalized to the time-point of gemcitabine addition and set to a value of $1 . \mathrm{IC}_{50}$ values were calculated at $72 \mathrm{~h}$.

PaCaDD-135, -159, -161, -165 and -188 and determined gene expression by qPCR (Table IB) after 3 and 6 days. Genes upregulated more than 2-fold compared to untreated cells were observed in the stem cell marker group (CD24, CD44, CD144 or EpCAM) in all 5 cell lines, in the stem cell associated gene group (PDX1, SHH, CBX7 or OCT4) in 4 of the 5 cell lines and in the EMT regulator group (SNAI1/Snail, SNAI2/Slug, TWIST) in all cell lines. While the pattern of upregulated individual genes varies among the cell lines, the only gene being upregulated in all cell lines under gemcitabine incubation was SNAI1/Snail, indicating a central role of EMT induction as a response to chemotherapy also in primary pancreatic cancer cell lines.

\section{Discussion}

In our study, we investigated the molecular alterations after 6 days of gemcitabine treatment in 2 distinct cell lines: the welldifferentiated Capan-1 (which was G1 in the original patient and shows G1 differentiation in nude or SCID mice xenografts) and the poorly differentiated Panc-1 cells (G3) $(31,32)$. We could show that cells surviving 6 days of continuous gemcitabine exposure express higher levels of almost all investigated stem cell markers, especially of PDX1, SHH, CD44, CD133 and Slug in Panc-1 cells and PDX1, OCT4, CD24, CD133, EpCAM and Snail in Capan-1 cells, respectively. The high expression of Slug in Panc-1 cells was accompanied by an increased migration in the scratch assay and by EMT-typical changes in the expression pattern of $\beta$-catenin and E-cadherin.

Efforts have been made to explain drug resistance based on the intrinsic detoxifying mechanisms of cancer stem cells. Some of the genes investigated here have been linked with chemoresistance in various models. One study investigating pancreatic cancer cell chemoresistance found that the repopulation of tumor cells following high-dose gemcitabine treatment was driven by $\mathrm{CD}_{4} 4^{+}$cells (33). Resistant pancreatic cancer cells cultured in the presence of gemcitabine with synchronous radiation therapy expressed increased levels of CD24, CD133, OCT4 and ABCG2 and showed phenotypic and molecular changes consistent with EMT (34). Consequently, their ability to migrate, form spheres and initiate tumors in nude mice was increased. In our study, we also show an increase in stem cell marker expression after 6 days of gemcitabine incubation. Most notably, even after only one day of treatment, PDX1, OCT4 and CD44 were already induced more than two-fold, indicating an induction in gene expression rather than selection of resistant cells. During organ development, PDX1 is expressed in epithelial cells in the foregut that give rise to the pancreatic buds and to the whole pancreas. Its expression is reactivated during the formation of pancreatic intraepithelial neoplasia (PanIN) and correlates with PanIN grade, is maintained in pancreatic cancer and affects patient survival (35). Similarly, in a study investigating OCT4 expression in pancreatic cancer samples, OCT4 expression was commonly found in metaplastic ducts (strong expression in $79.2 \%$ of cases), followed by pancreatic cancer cells (positive in $19.4 \%$ of cases) and lowest in non-tumorous pancreatic tissue (positive in $16.7 \%$ of cases) (36). These data indicate that stem cell associated genes are re-activated during tumor initiation. Our data indicate that OCT4 is induced early in tumor cell response to chemotherapeutic stimuli. 
An increasing number of studies show that there is a link between cancer stem cells and EMT. During development, $\mathrm{SHH}$ signals to the epithelial ventromedial somite wall and induces the connective tissue mesenchyme of the sclerotome, which is a physiological example of EMT (37), and is essential for forming three-dimensional structures from epithelial precursors. In our experiments, we see a strong increase of $\mathrm{SHH}$ accompanied by a strong increase of Slug in treated Panc-1 cells, and a weaker increase of SHH and Slug in Capan-1 cells. The downstream effector of the hedgehog pathway, Gli1, has been shown to induce the accumulation of $\beta$-catenin in the nucleus through Snail and E-cadherin (38). Loss of E-cadherin during EMT relocates $\beta$-catenin away from the cell membrane allowing individual cells to separate from cell clusters. We show that in response to gemcitabine, both Snail and Slug as well as $\mathrm{SHH}$ are overexpressed. Concordantly, we show the nuclear translocation of $\beta$-catenin and the loss of membraneous expression of E-cadherin, as expected in EMT. Further, EMT can also be regulated by Notch signaling, which is also increased in our experiments in Panc- 1 cells. While Snail is equally induced in Capan-1 and Panc-1 cells, Slug is differentially expressed between the two cell lines. This behavior could be explained by their respective baseline expression levels: Slug is expressed at low levels in Panc-1 cells and could thus be induced stronger compared Snail, which is expressed at higher baseline levels. Similar baseline expression levels of Snail and Slug have been shown by others both on transcript and protein levels (39). In cells surviving gemcitabine incubation, both EMT regulators are overexpressed thus indicating a protective role of this mechanism against the chemotherapeutic drug.

These data link the role of cells expressing stem cell markers to those undergoing EMT. Data obtained from experiments in benign mammary glands and mammary carcinomas show a direct link between EMT and the gain of epithelial stem-cell properties (25): in immortalized non-malignant human mammary epithelial cells, EMT was induced by treatment with TGF- $\beta$ or by ectopic expression of Snail or TWIST. As expected, these cells acquired a mesenchymal phenotype and a mRNA expression pattern consistent with EMT, and, most interestingly, exhibited a CD $44^{\text {high }} / \mathrm{CD} 24^{\text {low }}$ surface marker configuration - the antigenic phenotype ascribed to neoplastic mammary stem cells. This phenotype was also functionally associated with properties of stem cells as shown in their ability to form more mammospheres than untransformed cells.

Identifying a general marker or a set of markers to pinpoint stem cells in solid tumors has proven difficult, as exemplified by CD133, the expression of which has been used in the identification of cancer stem cells in various tumors, such as brain, lung, colon, kidney, prostate, liver, colon and skin cancer. However, its importance as a general 'stemness' marker has been questioned by recent studies on its expression in various normal glandular tissues in general $(40,41)$ and in normal adult pancreata and pancreatic ductal adenocarcinomas (42). In the healthy pancreas, CD133 expression is seen to be initiated in cells at the center of the acini soon after lumen formation, and continues along the ductal system into the following ducts up to the larger ducts, thereby fading in expression intensity. CD133 and CK19 (a ductal marker) are generally co-localized in the fully differentiated ductal epithelium. Although apical staining is seen in the majority of epithelial cells, cells located near the luminal surface within the acini showed strong cytoplasmic CD133 staining paired with CK19 negativity, indicating an altered differentiation. In tumor specimens, CD133 positive cells are identified in $80 \%$ of the cases, with mostly apical/endoluminal expression, and cytoplasmic staining is observed in about $1 \%$ of the malignant cells. In summary, in both normal pancreata and adenocarcinomas of the pancreas, there seem to exist two distinct populations of CD133 expressing cells: a major population with apical/endoluminal CD133 expression, which represents a particular stage in cell differentiation related to the formation of lumina and ducts, and a minor, rare, population which expresses CD133 in the cytoplasm. In our study we show that CD133 is enriched both on mRNA and protein levels in treated cells and particularly the treated Panc- 1 cells differ in their subcellular localization of CD133. Similar findings were reported from glioblastoma patients, which showed that CD133 positive cells were enriched in recurrent glioblastoma and showed increased resistance against a multitude of chemotherapeutic agents (43). While each of the putative stem cell markers CD24, CD44 or CD133 was increased in our two investigated established cell lines and partly in the primary cell lines as well, also other stem cell associated genes were upregulated. Along with the induction of these genes, downstream effectors of chemoresistance, such as ATP-binding cassette transporters could confer the resistant phenotype (33). While our experimental design does not allow to fully differentiate between gene induction and selection of putative cancer stem cells, based on our data we deduct that stem cell associated genes are an important factor in cellular response to chemotherapeutic agents such as gemcitabine. However, the role of each gene in chemoresistance and its downstream effectors has to be investigated carefully to elucidate a possible key gene, which might act as a therapeutic target.

\section{Acknowledgements}

The authors thank Astrid Taut and Isabel Zeitträger for their help in conducting the experiments. This study was in part funded by a grant of the 'Stiftung P. E. Kempkes' at the Philipps University of Marburg, Germany.

\section{References}

1. Ellison LF and Wilkins K: An update on cancer survival. Health Rep 21: 55-60, 2010.

2. Jemal A, Siegel R, Xu J and Ward E: Cancer statistics, 2010. CA Cancer J Clin 60: 277-300, 2010

3. Burris HA III, Moore MJ, Andersen J, et al: Improvements in survival and clinical benefit with gemcitabine as first-line therapy for patients with advanced pancreas cancer: a randomized trial. J Clin Oncol 15: 2403-2413, 1997.

4. Moore MJ, Goldstein D, Hamm J, et al: Erlotinib plus gemcitabine compared with gemcitabine alone in patients with advanced pancreatic cancer: a phase III trial of the National Cancer Institute of Canada Clinical Trials Group. J Clin Oncol 25: 1960-1966, 2007.

5. Conroy T, Paillot B, François E, et al: Irinotecan plus oxaliplatin and leucovorin-modulated fluorouracil in advanced pancreatic cancer - a Groupe Tumeurs Digestives of the Federation Nationale des Centres de Lutte Contre le Cancer study. J Clin Oncol 23: 1228-1236, 2005.

6. Ducreux M, Boige V and Malka D: Treatment of advanced pancreatic cancer. Semin Oncol 34: S25-S30, 2007.

7. Jemal A, Bray F, Center MM, et al: Global cancer statistics. CA Cancer J Clin 61: 69-90, 2011. 
8. Wang Z, Li Y, Ahmad A, et al: Pancreatic cancer: understanding and overcoming chemoresistance. Nat Rev Gastroenterol Hepatol 8: 27-33, 2011.

9. Klonisch T, Wiechec E, Hombach-Klonisch S, et al: Cancer stem cell markers in common cancers - therapeutic implications. Trends Mol Med 14: 450-460, 2008

10. Liu QH, Zhang J, Zhao CY, et al: Surviving cells after treatment with gemcitabine or 5-fluorouracil for the study of de novo resistance of pancreatic cancer. Cancer Lett 314: 119-125, 2012

11. Bonner-Weir S and Sharma A: Pancreatic stem cells. J Pathol 197: 519-526, 2002

12. O'Brien CA, Kreso A and Jamieson $\mathrm{CH}$ : Cancer stem cells and self-renewal. Clin Cancer Res 16: 3113-3120, 2010.

13. Zhang YQ, Kritzik $M$ and Sarvetnick N: Identification and expansion of pancreatic stem/progenitor cells. J Cell Mol Med 9: 331-344, 2005.

14. Lee CJ, Dosch J and Simeone DM: Pancreatic cancer stem cells. J Clin Oncol 26: 2806-2812, 2008.

15. Quante $M$ and Wang TC: Stem cells in gastroenterology and hepatology. Nat Rev Gastroenterol Hepatol 6: 724-737, 2009.

16. Wang JC and Dick JE: Cancer stem cells: lessons from leukemia. Trends Cell Biol 15: 494-501, 2005.

17. Zou GM: Cancer initiating cells or cancer stem cells in the gastrointestinal tract and liver. J Cell Physiol 217: 598-604, 2008.

18. Haraguchi $\mathrm{N}$, Utsunomiya $\mathrm{T}$, Inoue $\mathrm{H}$, et al: Characterization of a side population of cancer cells from human gastrointestinal system. Stem Cells 24: 506-513, 2006.

19. Haraguchi $\mathrm{N}$, Inoue $\mathrm{H}$, Tanaka F, et al: Cancer stem cells in human gastrointestinal cancers. Hum Cell 19: 24-29, 2006.

20. Hermann PC, Bhaskar S, Cioffi M and Heeschen C: Cancer stem cells in solid tumors. Semin Cancer Biol 20: 77-84, 2010.

21. Zhou J, Wang CY, Liu T, et al: Persistence of side population cells with high drug efflux capacity in pancreatic cancer. World J Gastroenterol 14: 925-930, 2008.

22. Wang Z, Li Y, Kong D, et al: Acquisition of epithelialmesenchymal transition phenotype of gemcitabine-resistant pancreatic cancer cells is linked with activation of the notch signaling pathway. Cancer Res 69: 2400-2407, 2009.

23. Arumugam T, Ramachandran V, Fournier KF, et al: Epithelial to mesenchymal transition contributes to drug resistance in pancreatic cancer. Cancer Res 69: 5820-5828, 2009.

24. Kim MP and Gallick GE: Gemcitabine resistance in pancreatic cancer: picking the key players. Clin Cancer Res 14: 1284-1285, 2008

25. Mani SA, Guo W, Liao MJ, et al: The epithelial-mesenchymal transition generates cells with properties of stem cells. Cell 133 704-715, 2008

26. Rückert F, Werner K, Aust D, et al: Immunohistological analysis of six new primary pancreatic adenocarcinoma cell lines. Global J Biochem 3: 11, 2012.

27. Rückert F, Aust D, Böhme I, et al: Five primary human pancreatic adenocarcinoma cell lines established by the outgrowth method. J Surg Res 172: 29-39, 2012.

28. Pfaffl MW, Horgan GW and Dempfle L: Relative expression software tool (REST) for group-wise comparison and statistical analysis of relative expression results in real-time PCR. Nucleic Acids Res 30: e36, 2002.
29. Pfaffl MW: A new mathematical model for relative quantification in real-time RT-PCR. Nucleic Acids Res 29: e45, 2001.

30. Ke N, Wang X, Xu X and Abassi YA: The xCELLigence system for real-time and label-free monitoring of cell viability. Methods Mol Biol 740: 33-43, 2011.

31. Sipos B, Moser S, Kalthoff H, et al: A comprehensive characterization of pancreatic ductal carcinoma cell lines: towards the establishment of an in vitro research platform. Virchows Arch 442: 444-52, 2003.

32. Neureiter D, Zopf S, Dimmler A, et al: Different capabilities of morphological pattern formation and its association with the expression of differentiation markers in a xenograft model of human pancreatic cancer cell lines. Pancreatology 5: 387-397, 2005.

33. Hong SP, Wen J, Bang S, Park S and Song SY: CD44-positive cells are responsible for gemcitabine resistance in pancreatic cancer cells. Int J Cancer 125: 2323-2331, 2009.

34. Du Z, Qin R, Wei C, et al: Pancreatic cancer cells resistant to chemoradiotherapy rich in 'stem-cell-like' tumor cells. Dig Dis Sci 56: 741-750, 2011

35. Quint K, Stintzing S, Alinger B, et al: The expression pattern of PDX-1, SHH, Patched and Gli-1 is associated with pathological and clinical features in human pancreatic cancer. Pancreatology 9: 116-26, 2009.

36. Wen J, Park JY, Park KH, et al: Oct4 and Nanog expression is associated with early stages of pancreatic carcinogenesis. Pancreas 39: 622-626, 2010.

37. Fan CM and Tessier-Lavigne M: Patterning of mammalian somites by surface ectoderm and notochord: evidence for sclerotome induction by a hedgehog homolog. Cell 79: 1175-1186, 1994.

38. Li X, Deng W, Lobo-Ruppert S and Ruppert J: Gli1 acts through Snail and E-cadherin to promote nuclear signaling by $\beta$-catenin. Oncogene 26: 4489-4498, 2007.

39. Hotz B, Arndt M, Dullat S, et al: Epithelial to mesenchymal transition: expression of the regulators Snail, Slug, and Twist in pancreatic cancer. Clin Cancer Res 13: 4769-4776, 2007.

40. Missol-Kolka E, Karbanová J, Janich P, et al: Prominin-1 (CD133) is not restricted to stem cells located in the basal compartment of murine and human prostate. Prostate 71 : 254-267, 2011

41. Karbanová J, Missol-Kolka E, Fonseca AV, et al: The stem cell marker CD133 (Prominin-1) is expressed in various human glandular epithelia. J Histochem Cytochem 56: 977-993, 2008.

42. Immervoll H, Hoem D, Sakariassen PØ, Steffensen OJ and Molven A: Expression of the 'stem cell marker' CD133 in pancreas and pancreatic ductal adenocarcinomas. BMC Cancer 8: 48, 2008

43. Liu G, Yuan X, Zeng Z, et al: Analysis of gene expression and chemoresistance of $\mathrm{CD} 133^{+}$cancer stem cells in glioblastoma. Mol Cancer 5: 67, 2006. 